\title{
Contribution of parvalbumin and somatostatin-expressing GABAergic neurons to slow oscillations and the balance in beta-gamma oscillations across cortical layers
}

\section{Toshinobu Kuki ${ }^{1}$, Kazuyuki Fujihara ${ }^{2,3,4}$, Hideki Miwa ${ }^{2,4}$, Nobuaki Tamamaki ${ }^{5}$, Yuchio Yanagawa ${ }^{2,4}$ and Hajime Mushiake ${ }^{1,4 *}$}

${ }^{1}$ Department of Physiology, Graduate School of Medicine, Tohoku University, Sendai, Japan

${ }^{2}$ Department of Genetic and Behavioral Neuroscience, Gunma University Graduate School of Medicine, Maebashi, Japan

${ }^{3}$ Department of Psychiatry and Human Behavior, Gunma University Graduate School of Medicine, Maebashi, Japan

${ }^{4}$ Core Research for Evolutional Science and Technology, Tokyo, Japan

${ }^{5}$ Department of Morphological Neural Science, Graduate School of Medical Sciences, Kumamoto University, Kumamoto, Japan

\section{Edited by:}

Yoshiyuki Kubota, National Institute for Physiological Sciences, Japan

\section{Reviewed by:}

Katsunori Kitano, Ritsumeikan

University, Japan

M. Victoria Puig, IMIM-Hospital del Mar Medical Research Institute, Spain

\section{*Correspondence:}

Hajime Mushiake, Department of Physiology, Graduate School of Medicine, Tohoku University, 2-1, Aoba-Ku, Sendai 980-8575, Japan e-mail: hmushiak@med.tohoku.ac.jp
Cortical interneurons are classified into several subtypes that contribute to cortical oscillatory activity. Parvalbumin (PV)-expressing cells, a type of inhibitory interneuron, are involved in the gamma oscillations of local field potentials (LFPs). Under ketamine-xylazine anesthesia or sleep, mammalian cortical circuits exhibit slow oscillations in which the active-up state and silent-down state alternate at $\sim 1 \mathrm{~Hz}$. The up state is composed of various high-frequency oscillations, including gamma oscillations. However, it is unclear how PV cells and somatostatin (SOM) cells contribute to the slow oscillations and the high-frequency oscillations nested in the up state. To address these questions, we used mice lacking glutamate decarboxylase 67, primarily in PV cells (PV-GAD67 mice) or in SOM cells (SOM-GAD67 mice). We then compared LFPs between PV-GAD67 mice and SOM-GAD67 mice. PV cells target the proximal regions of pyramidal cells, whereas SOM cells are dendrite-preferring interneurons. We found that the up state was shortened in duration in the PV-GAD67 mice, but tended to be longer in SOM-GAD67 mice. Firing rate tended to increase in PV-GAD67 mice, but tended to decrease in SOM-GAD67 mice. We also found that delta oscillations tended to increase in SOM-GAD67 mice, but tended to decrease in PV-GAD67 mice. Current source density and wavelet analyses were performed to determine the depth profiles of various high-frequency oscillations. High gamma and ripple $(60-200 \mathrm{~Hz})$ power decreased in the neocortical upper layers specifically in PV-GAD67 mice, but not in SOM-GAD67. In addition, beta power $(15-30 \mathrm{~Hz})$ increased in the deep layers, specifically in PV-GAD67 mice. These results suggest that PV cells play important roles in persistence of the up state and in the balance between gamma and beta bands across cortical layers, whereas SOM and PV cells may make an asymmetric contribution to regulate up-state and delta oscillations.

Keywords: PV cells, slow oscillation, gamma oscillation, beta oscillation, neocortex, CSD, wavelet analysis, mouse

\section{INTRODUCTION}

Parvalbumin (PV)-expressing cells are the major type of inhibitory gamma-aminobutyric acid-producing (GABAergic) interneurons of the neocortex. These cells are distributed across cortical layers, target the proximal regions of pyramidal cells, and are characterized by a fast-spiking physiological phenotype (Kawaguchi, 1993; Kawaguchi and Kubota, 1993; Condé et al., 1994; Tamamaki et al., 2003). In contrast, somatostatin (SOM)expressing cells are dendrite-preferring interneurons (Martinotti, 1889; Fairèn et al., 1984; Somogyi and Cowey, 1984). PV cells are involved in generating gamma $(\gamma)$ oscillations in local field potentials (LFPs) (Freund, 2003; Fuchs et al., 2007; Sohal et al., 2009; Buzsáki and Wang, 2012). Gamma oscillations are found in the upper layers in particular (Roopun et al., 2006).
During ketamine-xylazine anesthesia or sleep, slow oscillations occur in the cortex (Steriade et al., 1993a,b,c; Destexhe et al., 1999; Timofeev et al., 2000). A slow oscillation is an alternation between the active up state and silent down state at $\sim 1 \mathrm{~Hz}$ (Wilson and Kawaguchi, 1996; Anderson et al., 2000; Kitano et al., 2002), and also triggers and groups delta oscillations $(1-4 \mathrm{~Hz}$; Amzica and Steriade, 1998). Various high-frequency oscillations, including $\gamma$ oscillations, are nested within the up state (Steriade et al., 1996; Hasenstaub et al., 2005; Ruiz-Mejias et al., 2011). The up state is an intriguing model of high-frequency activity, which is a selforganized ensemble of neurons maintaining balanced excitatory and inhibitory inputs.

Many studies have investigated the up state mechanism. The up state is generated by deep pyramidal neurons and 
interneurons, including PV cells. Deep-layer 5 pyramidal neurons fire earlier than neurons in other layers during the up state (Sanchez-Vives and McCormick, 2000). Layer-specific inhibition studies show that deep layer pyramidal neurons are necessary during the up state (Wester and Contreras, 2012; Beltramo et al., 2013). Fast-spiking interneurons may initiate the up state via thalamo-cortical inputs (Puig et al., 2008; Ushimaru et al., 2012). Pacemaker-like Martinotti cells in layer 5 may also initiate the up state (Le Bon-Jego and Yuste, 2007). Furthermore, a $G A B A(A)$ receptor antagonist shortens up state duration, suggesting that GABAergic interneurons help maintain the up state (Mann et al., 2009; Sanchez-Vives et al., 2010). In addition, a study of patients with schizophrenia demonstrated that GABAsynthesizing enzyme glutamate decarboxylase 67 (GAD67) content is lower in PV cells, and that their network activity (i.e., $\gamma$ oscillations) is impaired, suggesting a link between GAD67 in PV cells and $\gamma$ oscillations (Lisman et al., 2008). GABAergic circuits including PV cells also develop multisensory integration in the insular cortex, a deficiency of which may be relevant to schizophrenia and autism (Gogolla et al., 2014).

However, it remains unclear how interneuronal subtypes contribute to the persistence of the up state and high-frequency oscillations within the up state. Therefore, we propose two hypotheses. The first is that PV cells contribute to maintain the up state. The second is that PV cells are also involved in balancing the high-frequency oscillations across cortical layers. To test these hypotheses, we examined the up state of slow oscillations and high frequencies using mice lacking GAD67 in PV or SOM cells. We recorded LFPs along the cortical layers of knockout mice under ketamine and xylazine anesthesia. We also applied wavelet transformation to the CSD data (Lakatos et al., 2005; Maier et al., 2011).

\section{MATERIALS AND METHODS ANIMALS}

All animal experiments were approved by the Tohoku University Committee for Animal Research, The Animal Care and Experimentation Committee of Gunma University, Showa Campus, and The Animal Research Committee of Kumamoto University. Every effort was made to minimize the number of animals used and their suffering. To achieve a homozygous deletion of the GAD67 gene primarily in PV cells or in SOM cells, GAD67-floxed mice (Obata et al., 2008) were crossed either with PV-Cre mice expressing Cre recombinase (Cre) under control of a bacterial artificial chromosome transgenic PV promoter fragment (Tanahira et al., 2009) or with SOM-IRES-Cre mice expressing Cre under control of an endogenous SOM promoter (Taniguchi et al., 2011). We refer to the GAD67 $7^{\text {flox/flox} / P V-C r e ~ m i c e, ~ t h e ~ G A D 67 ~ f l o x / f l o x / S O M ~}{ }^{\text {IRES-Cre/+ }}$ mice, and the GAD67 flox/flox mice as PV-GAD67 mice, SOMGAD67 mice, and control mice, respectively, hereafter. The mice were of either sex. The PV-GAD67 mice and control mice were littermates, and the SOM-GAD67 mice and their control mice were also littermates.

\section{SURGICAL PROCEDURES}

Surgical anesthesia was induced by intraperitoneal injection of ketamine hydrochloride $(100 \mathrm{mg} / \mathrm{kg})$ and xylazine $(10 \mathrm{mg} / \mathrm{kg})$.
All surgical or pressure points were treated with $0.25 \%$ lidocaine hydrochloride. The anesthesia plane for the surgery was determined by the absence of a vibrissa motor response, a leg withdrawal response, and an eye-blink reflex. Additional doses of the ketamine and xylazine mixture were injected intraperitoneally, as required. After placing the animal on a stereotaxic apparatus, a small craniotomy and duratomy were made above the somatosensory area $(1.5 \mathrm{~mm}$ lateral and $1.6 \mathrm{~mm}$ rostral from bregma) on the right hemisphere to insert the recording electrode. The coordinates were determined according to Franklin and Paxinos (2008). A skull screw was placed above the cerebellar cortex of the left hemisphere as a voltage reference.

\section{ELECTROPHYSIOLOGICAL RECORDINGS}

Electrophysiological recordings were taken using single-shank electrodes with a linear array of 16 recording channels (16 channels, $100-\mu \mathrm{m}$ inter channel spacing; A1 × 16-10 mm, 100-413; Neuronexus, Inc., Ann Arbor, MI, USA). The distance between the two channels was $100 \mu \mathrm{m}$. The impedance of the probes was $\sim 0.7 \mathrm{~mW}$. The signals were amplified at unity gain using a head stage amplifier (HST/8o50-G1-GR; Plexon, Inc., Dallas, TX, USA) and were further amplified (gain: $250 \times$ ) and recorded using a 16-channel recording system (Omniplex; Plexon) at a $40-\mathrm{kHz}$ sampling rate. Then, the signal was split into LFP $(\sim 200 \mathrm{~Hz})$ and multi-unit activity (MUA; $\sim 300 \mathrm{~Hz}$ ) ranges by analog filtering. LFP was down-sampled at $1000 \mathrm{~Hz}$. We recorded the signal in each animal for about $30 \mathrm{~min}$. Stable representative data used for all analyses were defined as those between anaesthesias for about $1 \mathrm{~min}$ in each animal (about 30 up states in each animal). Total data used for analysis was about $20 \mathrm{~min}$.

\section{PERFUSION AND HISTOLOGY}

After the recording session, we electrified the recording sites using a multi-channel probe to mark probe position. Next, $5 \mu \mathrm{A}$ of electricity was passed until the sum of electricity reached $400 \mu \mathrm{C}$. The mice were then euthanized with intraperitoneal pentobarbital $(120 \mathrm{mg} / \mathrm{kg})$. After voluntary breathing stopped, the mice were transcardially perfused with phosphate buffer $(\mathrm{PB} ; 0.4 \mathrm{M}$, $\mathrm{pH} 7.4$ ), followed by $100 \mathrm{~mL} \mathrm{4 \%}$ formaldehyde (FA) in PB. The whole brain was removed and post-fixed in 4\% FA overnight at $4^{\circ} \mathrm{C}$. After cryoprotection using a graded series of sucrose solutions $(10,20$, and $30 \%$ in $\mathrm{PB})$ at $4^{\circ} \mathrm{C}$, each brain specimen was frozen using compressed $\mathrm{CO}_{2}$ and sliced at $40 \mu \mathrm{m}$ using a rotary microtome (TU-213; Yamato Kohki Industrial, Saitama, Japan). The slices were mounted on gel-coated slides (MASGPtypeA S9901 white; Matsunami Glass Ind., Ltd., Osaka, Japan), dried, and stained using cresyl echt violet and coverslipped. The stained slides were inspected and photographed microscopically (BZ-9000; Keyence, Osaka, Japan) to verify the recording loci.

\section{DATA ANALYSIS}

Wavelet analyses were performed using a custom-written program in R (using function cwt() in package "Rwave"). Briefly, the LFP recording, $f(t)$, was transformed as follows (Mizuhara and Yamaguchi, 2011):

$$
W(b, a)=\frac{1}{\sqrt{a}} \int_{-\infty}^{\infty} f(t) G\left(\frac{t-b}{a}\right),
$$


where $a, b$ denote the scaling factor $(1 / \mathrm{Hz})$ and the center location (ms) of the mother wavelet function, respectively. $1 / a$ varied from 0.1 to $500 \mathrm{~Hz}$.

In Equation (1), $G(x)$ is the complex Gabor function:

$$
G(x)=\frac{1}{\sqrt{2 \pi}} \exp \left(-\left(\frac{x}{2 F_{s}}\right)^{2}\right) \exp \left(\frac{2 \pi i}{F s} x\right),
$$

where $F s$ is the sampling rate $(1000 / \mathrm{s})$.

In Figures 2, 5, the wavelet power spectrum (WPS), $|W(b, a)|$, from each LFP recording is shown. The WPS was scaled linearly from zero to one and color-coded. In Figures 5, 6, the WPS was sorted according to each high frequency band: alpha (7$14 \mathrm{~Hz})$, beta $(15-30 \mathrm{~Hz})$, low gamma $(30-60 \mathrm{~Hz})$, high gamma $(60-90 \mathrm{~Hz})$, and ripple $(100-200 \mathrm{~Hz})$ bands. To detect the up state, the mean of all channel WPS across high frequency bands $(10-100 \mathrm{~Hz}$ ) was used (semi-transparent red color in Figures 2, 4, 5). To reduce any fluctuations unrelated to the up state, we applied simple moving mean to WPS:

$$
M(t)=\sum_{T=t-\frac{n}{2}}^{t+\frac{n}{2}} W P S(T) /(n * F s+1),
$$

where $n$ is the width of the simple moving mean $(0.25 \mathrm{~s}), F s$ is the sampling rate $(1000 / \mathrm{s})$, and $t$ is the central time. We set the state criteria as half the power of the largest $M(t)$ (mean of top 5\%). We determined those $M(t)$ with a spectral power larger than the criteria to be up state and the others as down state. The mean trigger of the up state was calculated according to the $400 \mathrm{~ms}$ before and after the up state (Figures 4B,D,F, 6B,D,F). All data from each animal were averaged at the start of the up state for the statistical analyses.

For CSD analysis, each of the LFP recordings was filtered across channels using Hanning windowing to remove high-frequency noise (Rappelsberger et al., 1981):

$$
\overline{f_{n}}(t)=0.23 f_{n-1}(t)+0.54 f_{n}(t)+0.23 f_{n+1}(t),
$$

where $f_{n}(t)$ is the field potential recorded at the $n$th channel.

The following formula was used to obtain the (relative) CSD (Nicholson and Freeman, 1975; Mitzdorf and Singer, 1977; Rappelsberger et al., 1981; Chauvette et al., 2010):

$$
\bar{I}_{n}(t)=-\left(\bar{f}_{n-1}(t)-2 \bar{f}_{n}(t)+\bar{f}_{n+1}(t)\right) .
$$

In this formula, the resistance of the neural tissue was assumed to be constant across the cortical layer, and the other physical constants were ignored for simplicity. Relative WPS of CSD was calculated by measuring the ratio of the WPS within the band of interest to the total power of the high frequency bands (from alpha to ripple) within each channel (Figures 5, 6; Carlén et al., 2011).

\section{STATISTICAL ANALYSIS}

$N$ always refers to the number of animals. Welch's two-sample $t$-tests were used together with the Benjamini-Hochberg correction. The normalized values are shown in Figure 3, separated by values for the control mice to increase clarity. The differences in Figure 6 were calculated by subtracting the values for knockout mice from those for the control mice. All statistical tests are based on data without subtraction or normalization.

\section{RESULTS \\ DEPTH PROFILE OF SLOW OSCILLATIONS ACROSS THE CORTICAL LAYERS}

We recorded LFPs across the cortical layers during slow oscillations under ketamine anesthesia using a 16-channel silicon probe (Figures 1A,B). Our region of interest was the neocortex, although the probe tip reached the hippocampus underneath the neocortex. The slow oscillations were characterized by synchronous oscillation $(<1 \mathrm{~Hz})$ throughout the corticohippocampus, whereas polarity reverses with depth of the cortical layer (Steriade et al., 1993a,b; Chauvette et al., 2010). In addition, the high-frequency power was stronger in the area around the hippocampus than that near the neocortex (Wolansky et al., 2006; Sharma et al., 2010).

\section{SELECTIVE DECREMENT OF THE UP STATE DURATION IN PV-GAD67 MICE}

We investigated the role of PV-expressing cells (PV cells) in cortical oscillatory activity using PV-GAD67 mice and SOM-GAD67 mice in which GAD67 was ablated primarily in PV or SOM cells, respectively. We first assessed the effects of the functional deficiency on slow oscillations between PV and SOM cells, and then compared the state durations and firing rates of PV-GAD67 and SOM-GAD67 mice with those of control mice. We detected up and down states by quantitatively evaluating the high-frequency power $(10-100 \mathrm{~Hz})$ of LFPs using wavelet analyses (Figure 2). Most of the multi-unit activity (MUA) was concomitant with detecting the up state. Although the delta oscillations of at about $2 \mathrm{~Hz}$ were contaminated, the strong WPS of the slow oscillation up states was detected separately from the weak WPS of

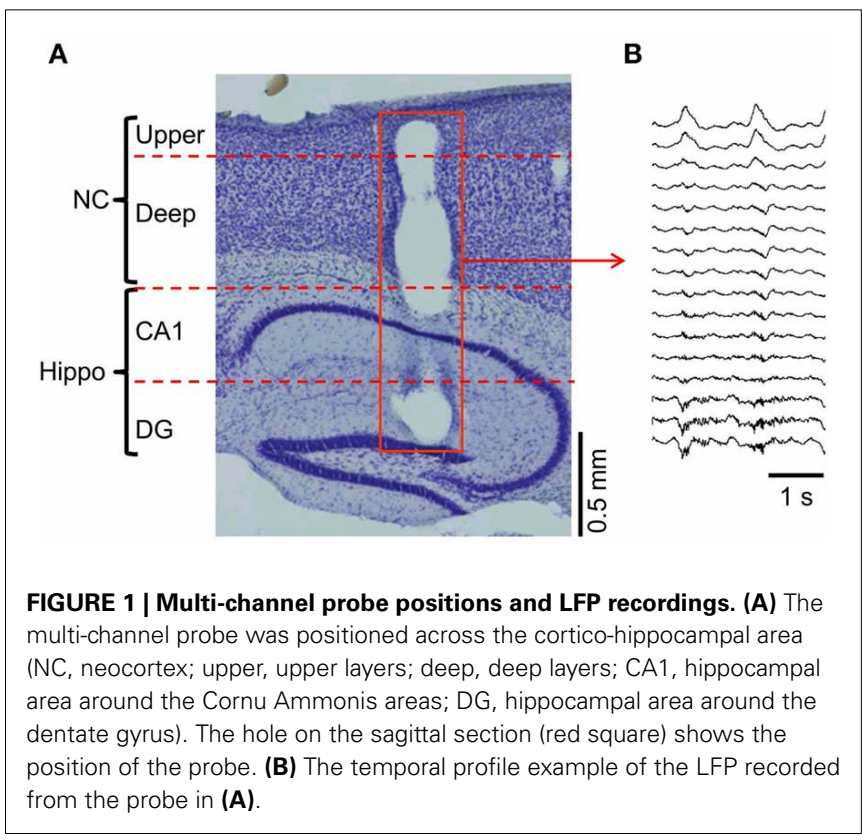


A

\section{Control}

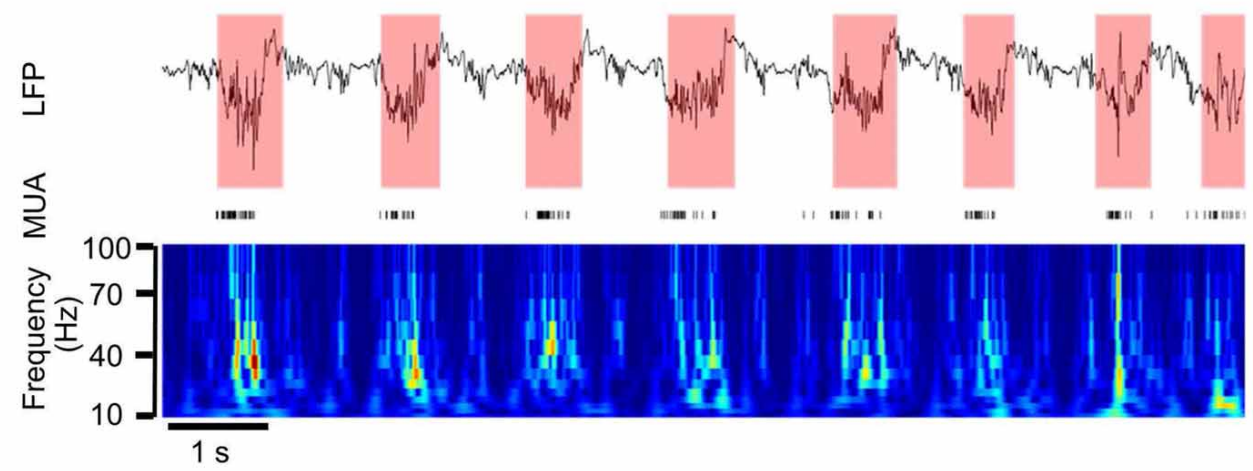

B

PV-GAD67

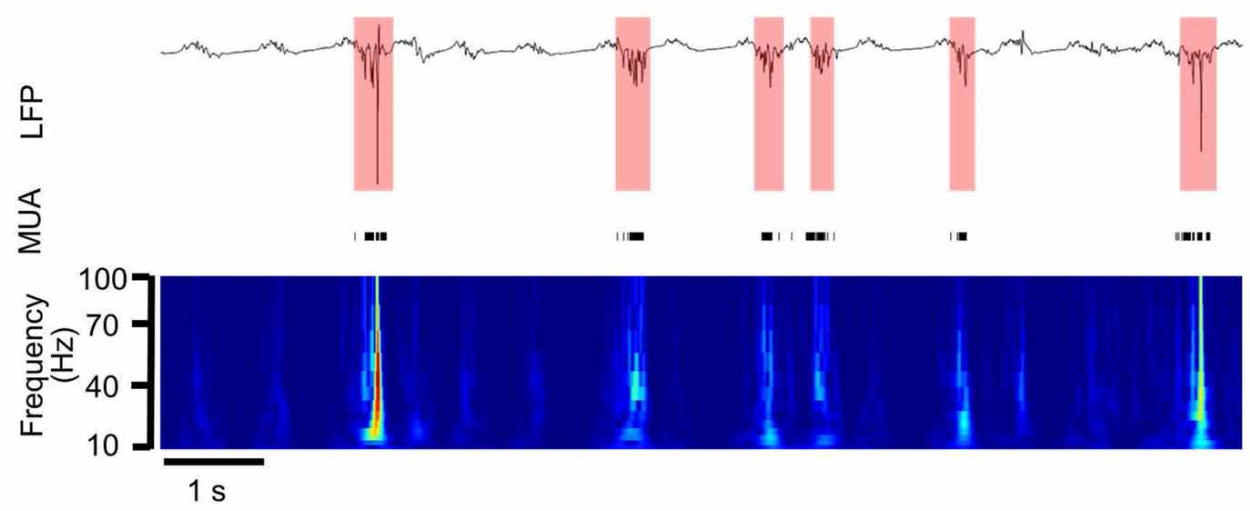

C

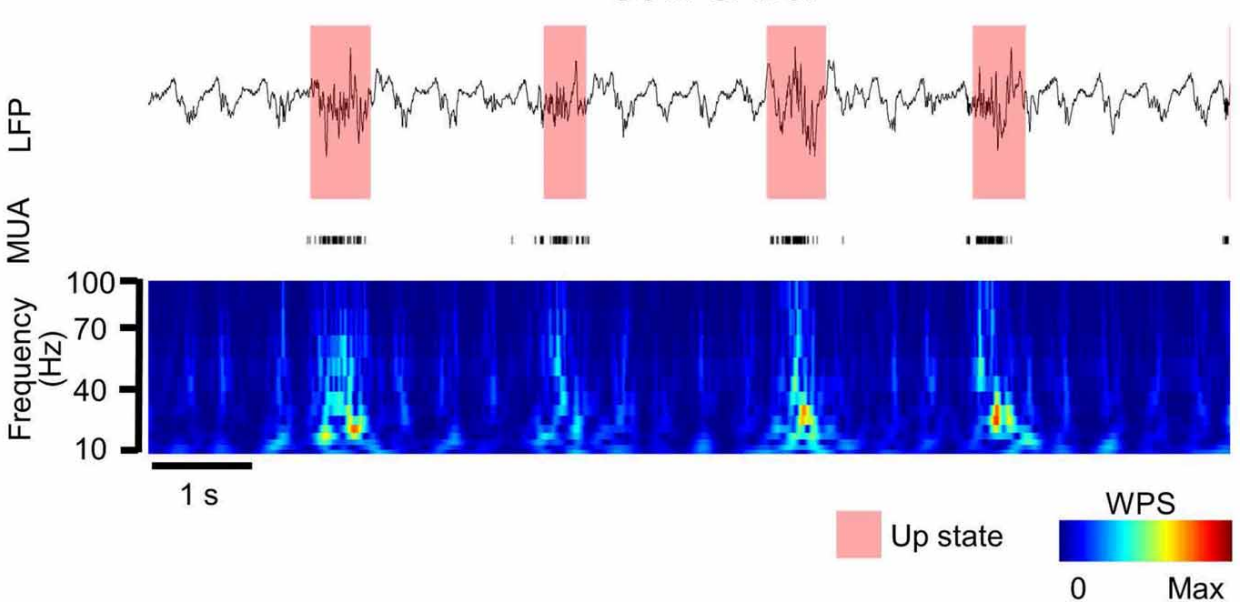

FIGURE 2 | LFP and MUA temporal profile and the up state detected from the WPS of the LFP. (A) The LFP temporal profile example from channel 14 (top), the MUA temporal profile from all channels (middle), and the WPS (bottom) of a control mouse (semi-transparent red, up state detected from the WPS; colored bar, normalized amplitude of WPS). (B) PV-GAD67 mouse under the same conditions described in (A). The up state tended to be shorter and the down state longer in duration compared with the control mouse. (C) SOM-GAD67 mouse under the same conditions described in (A). The down state tended to be longer in duration compared with that in the control mouse. the delta oscillation. Therefore, we detected the up state containing a stronger high-frequency power than that for the down state. We compared control mice (Figure 2A) and PV-GAD67 mice (Figure 2B) in terms of state duration. PV-GAD67 mice displayed a shorter up state and a longer down state compared with control mice. Next, we compared control mice (Figure 2A) and SOM-GAD67 mice (Figure 2C) in terms of state duration. SOM-GAD67 mice displayed a longer down state compared with control mice. Our statistical analysis revealed that the up state of PV-GAD67 mice, but not SOM-GAD67 mice, was shorter in 
duration than that of control mice (Welch's two-sample $t$-test; $p=2.3 \times 10^{-3} ; N=5$; Figure 3A). The down state tended to be of longer duration in PV-GAD67 and SOM-GAD67 mice than in control mice, although not significantly (Welch's twosample $t$-test, Figures 2, 3B). Although the variability in down state duration appeared to be larger in SOM-GAD67 than in PVGAD67 mice, no statistical difference was observed between them (Welch's two-sample $t$-test; $N=5,4$; Figure 3B). To investigate the relationship between MUA and up state duration, we also compared firing rate of MUA during the up state in PV-GAD67 and SOM-GAD67 mice with that in control mice (Figure 3C). The high firing rates with GABA antagonists contributed to shorter up state (Sanchez-Vives et al., 2010). We found that firing rate tended to increase in PV-GAD67 mice, whereas it tended to decrease in SOM-GAD67 mice. However, these differences were not significant (Welch's two-sample $t$-test; $N=5$, 4 ; Figure 3C).

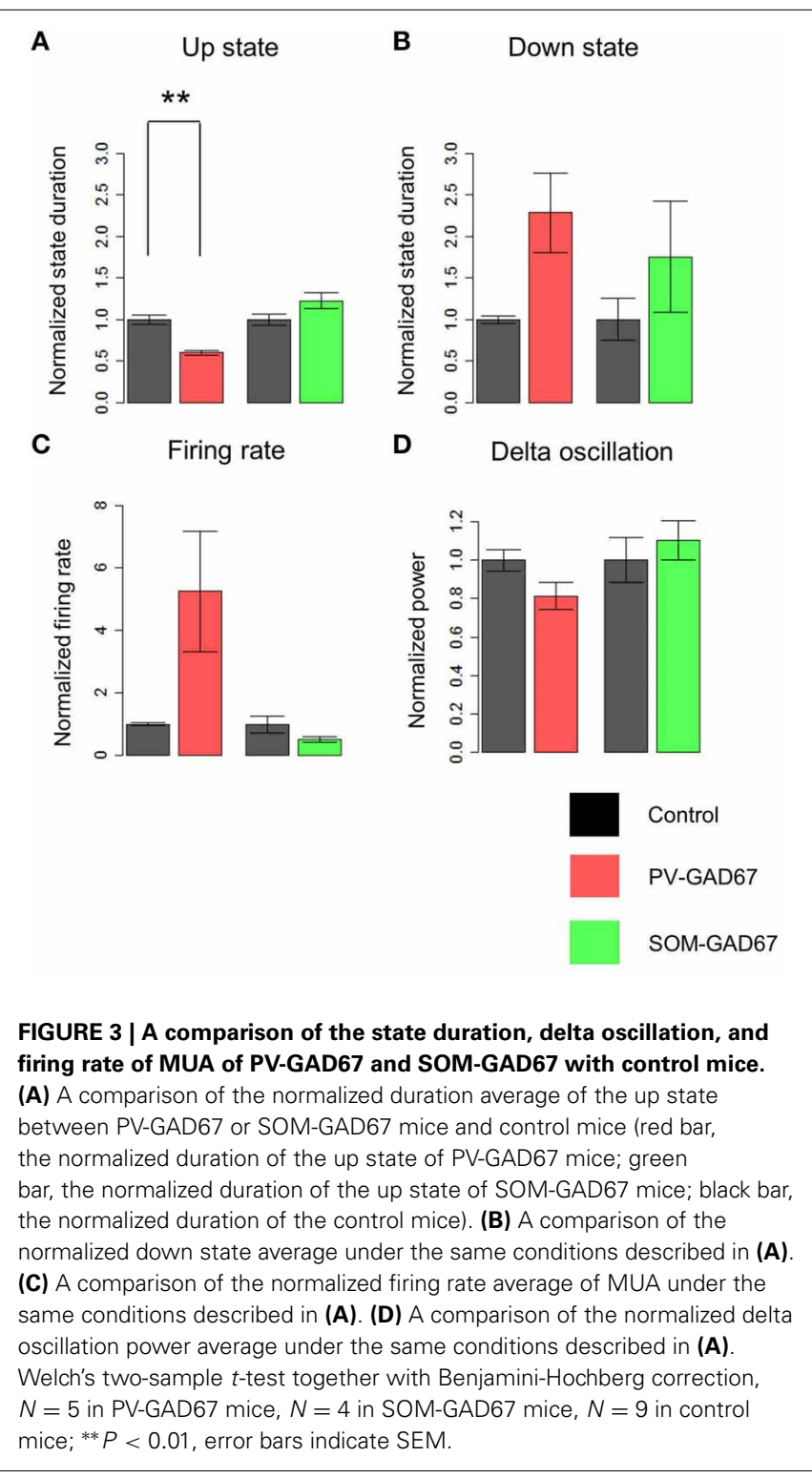

Therefore, the up state, but not the down state, was shortened specifically in PV-GAD67 mice. MUA during the up state tended to change asymmetrically between PV-GAD67 and SOM-GAD67 mice.

\section{ASYMMETRIC ALTERNATION OF DELTA OSCILLATION IN SOM-GAD67 AND PV-GAD67 MICE}

The delta oscillations $(1-4 \mathrm{~Hz})$ in Figure 2, co-occurring with slow oscillations, appeared relatively stronger in SOM-GAD67 than in PV-GAD67 and control mice. To investigate the effects of inhibitory subtypes on delta oscillations, we compared the power of delta oscillations across mice strains (Figure 3D). Delta oscillation power tended to increase in SOM-GAD67 mice, whereas it tended to decrease in PV-GAD67 mice, although these tendencies were not significant (Welch's two-sample $t$-test; $N=$ $5,4)$. However, delta oscillation power tended to asymmetrically alternate between PV-GAD67 and SOM-GAD67 mice.

\section{CSD ANALYSIS OF LFPs DURING THE UP STATE}

We conducted a CSD analysis before investigating the origin of LFP oscillations with wavelet analysis and detected the sink and source patterns across the cortical layers. A CSD analysis removes volume conduction (i.e., leakage of electrical fields from an electrical primary current source through biological tissue toward measurement sensors). The sink is the inward current, which is mainly caused by excitatory postsynaptic potentials, and the source is the outward current, which is mainly caused passively by the sink (Mitzdorf, 1985). Our CSD profile examples in the control, PV-GAD67, and SOM-GAD67 mice are shown in Figures 4A,C,E. To eliminate noise, we averaged the CSD profile triggered at the start of the up state (Figures 4B,D,F). We found a sink in the neocortical deep layers and a source in the upper layers in control and PV-GAD67 mice. We found a sink in CA1 and a source in the dentate gyrus of the hippocampus. We also found a weak sink in neocortical deep layers although a strong sink in neocortical upper layers in SOM-GAD67 mice. These results are consistent with other studies. The neocortical CSD in the up state becomes the source in the upper layers and the sink in deep layers (Chauvette et al., 2010). In contrast, the hippocampal CSD of the up state becomes the source in areas around the CAl and the sink around the dentate gyrus (Wolansky et al., 2006; Sharma et al., 2010).

\section{HIGH FREQUENCY OSCILLATORY DEPTH PROFILES OF THE UP STATE IN INTERNEURON SUBTYPE GAD67 KNOCKOUT MICE}

To investigate the depth profile of high frequencies across the cortical layers, we performed time-frequency analysis (wavelet analysis) of the CSD depth profiles (Lakatos et al., 2005; Maier et al., 2011). We classified the WPS into five frequency bands: alpha $(7-14 \mathrm{~Hz})$, beta $(15-30 \mathrm{~Hz})$, low gamma $(30-60 \mathrm{~Hz})$, high gamma $(60-90 \mathrm{~Hz})$, and ripple $(100-200 \mathrm{~Hz})$ bands. Here, we show a depth profile example consisting of five high-frequency bands in control, PV-GAD67, and SOM-GAD67 mice (Figures 5A,C,E). We also averaged the WPS aligned with the onset of the up state in the control, PV-GAD67, and SOM-GAD67 mice examples (Figures 5B,D,F). The PV-GAD67 and SOM-GAD67 mice examples exhibited many differences across layers compared with 


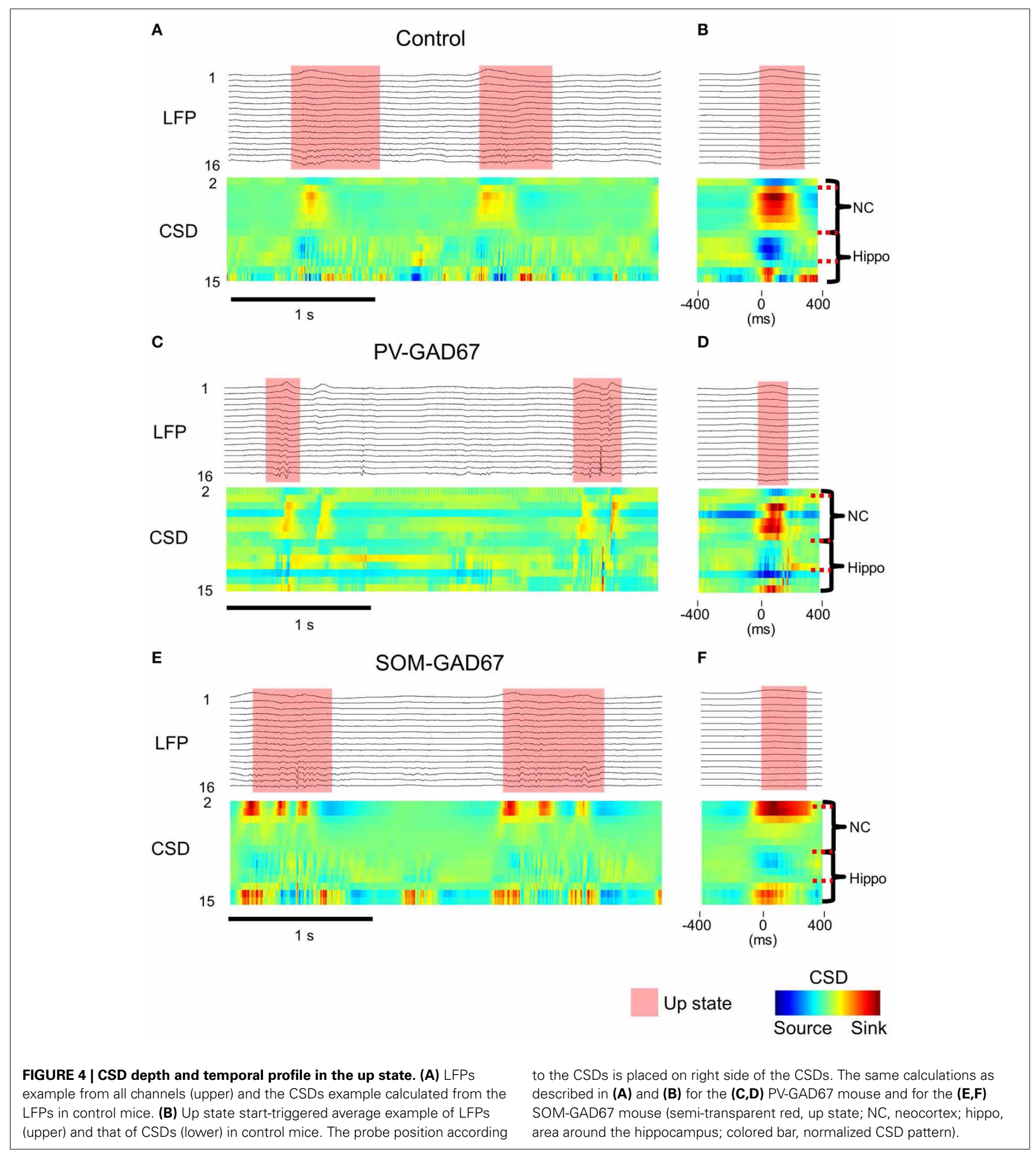

control mice example. For example, decreases in gamma and ripple in neocortical upper layers and increases in beta and low gamma in neocortical deep layers were observed in PV-GAD67 mice example (Figures 5B,D). Ripple and high gamma increased in the cortical upper layers, whereas low gamma increased in the neocortex, and beta decreased in the cortical deep layers of SOM-GAD67 mice example. We then calculated the difference in WPS between control and PV-GAD67 or SOM-GAD67 mice (Figures $6 \mathrm{~A}, \mathrm{~B})$. This population analysis revealed that part of these differences in WPS was reproduced. The high gamma and ripple power decreased in PV-GAD67 mice compared with control mice, particularly in the upper neocortical layers. Beta 


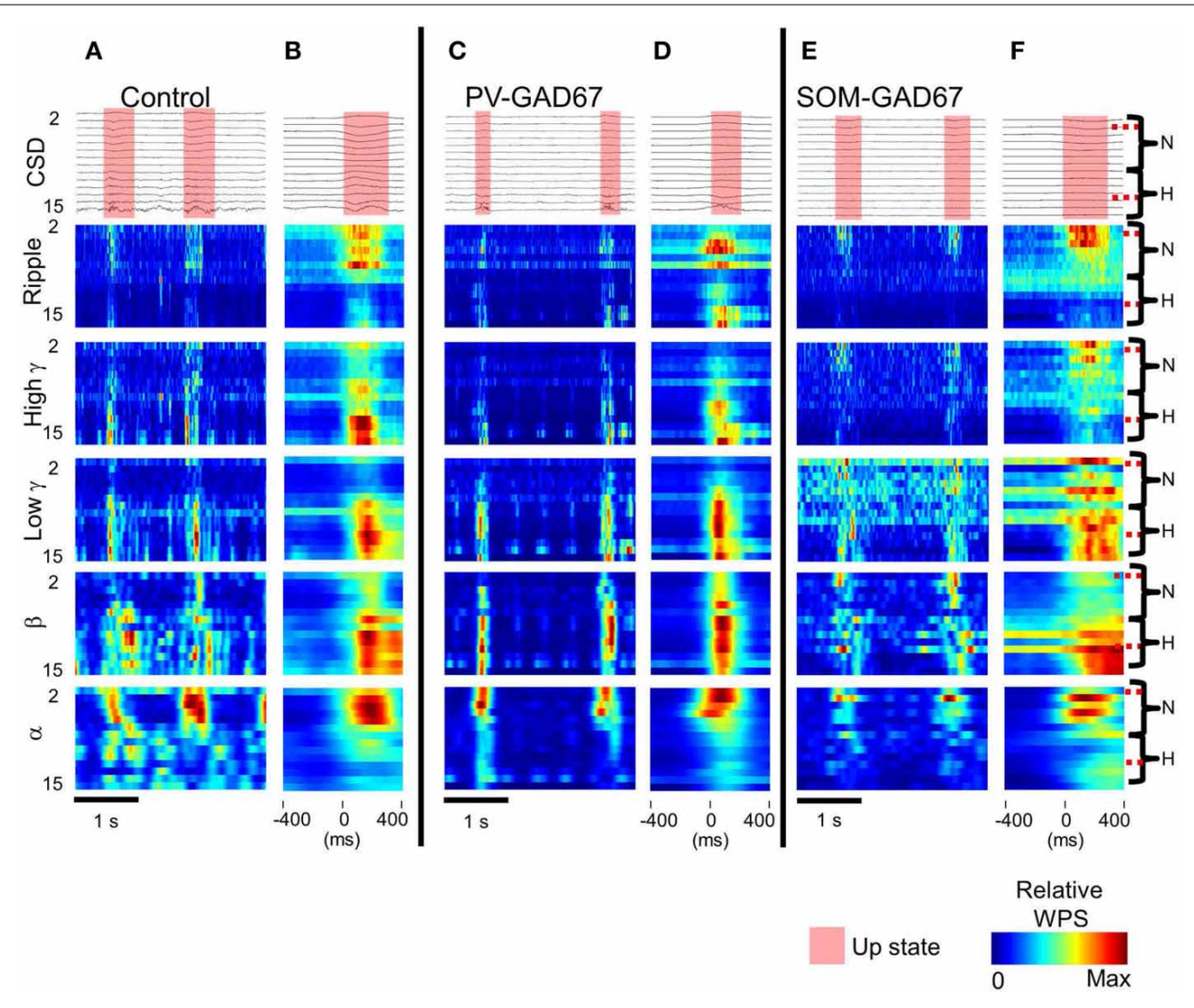

FIGURE 5 | The CSD WPS depth profiles in each high frequency band.

(A) CSDs (top) and WPS example calculated from the CSDs sorted according to each high frequency band (from the bottom alpha, 7-14 Hz; beta, $15-30 \mathrm{~Hz}$; low gamma, 30-60 Hz; high gamma, $60-90 \mathrm{~Hz}$; ripple, $100-200 \mathrm{~Hz}$ ) in control mice. (B) Up state start-triggered average of CSDs (upper) and of WPSs (lower five rows) in control mice. $\mathrm{N}$, neocortex; $\mathrm{H}$, region around the hippocampus; semi-transparent red, up state; color bar, relative WPS. (C) The same as A, but for PV-GAD67 mice. (D) The same as (B), but for PV-GAD67 mice. (E) The same as A, but for SOM-GAD67 mice. (F) The same as (B), but for SOM-GAD67 mice. power also increased in PV-GAD67 mice, particularly in the deep neocortical layers. These changes were also shown in the examples (Figures 5B,D). In contrast, SOM-GAD67 mice showed little change compared with control mice. Little change was observed between the PV-GAD67 mice or SOM-GAD67 mice and control mice in the area around the hippocampus. Next, we tested these changes statistically (Figure 6B). The statistical analysis revealed that the high gamma and ripple powers decreased significantly in the neocortex of the PV-GAD67 mice. In addition, beta power in the neocortex increased significantly in PV-GAD67 mice (Welch's two sample $t$-test; $N=5$; high $\gamma$, ripple, and beta; Figure 6B, upper). In contrast, no significant differences were observed between SOM-GAD67 and control mice (Welch's twosample $t$-test; $N$ - 4; Figure 6B, lower). Therefore, the gamma and ripple power decreased, particularly in the upper layers, and beta power increased, particularly in the deep layers in PV-GAD67 mice, whereas few changes were observed in SOM-GAD67 mice.

\section{DISCUSSION}

Our results demonstrate that up state duration of the slow oscillations was shortened in PV-GAD67 mice, but not in SOMGAD67 mice. Furthermore, high gamma $(60-90 \mathrm{~Hz})$ and ripple
$(100-200 \mathrm{~Hz})$ oscillation power decreased in the shortened up state, particularly in the neocortical upper layers, whereas beta oscillation $(15-30 \mathrm{~Hz})$ power increased in the neocortical deep layers. Delta oscillation power tended to increase in SOM-GAD67 mice, whereas it tended to decrease in PV-GAD67 mice. These results strongly suggest that $\mathrm{PV}$ cells specifically maintain the up state, high gamma and ripple oscillations in the upper layers and suppress beta oscillations in the deep layers. They also suggest that SOM and PV cells regulated delta oscillations.

A shortened up state in PV-GAD67 mice and a tendency for an increase in firing rate during the up state are consistent with previous studies using GABA antagonists. A GABA(A) antagonist induced a parametric shortening of up state duration in a dose-dependent manner. Cellular activities during the shortened up state, such as the firing rate, also increases (Mann et al., 2009; Sanchez-Vives et al., 2010). However, the involvement of GABAergic interneuronal subtypes in shortening of the upstate has not been shown. An important question is how PV-GAD67 mice, but not SOM-GAD67 mice, shorten their up state. Functional and anatomical differences exist between PV and SOM cells. PV cells inhibit the cell body and proximal dendrites of pyramidal cells, whereas SOM cells inhibit the distal 


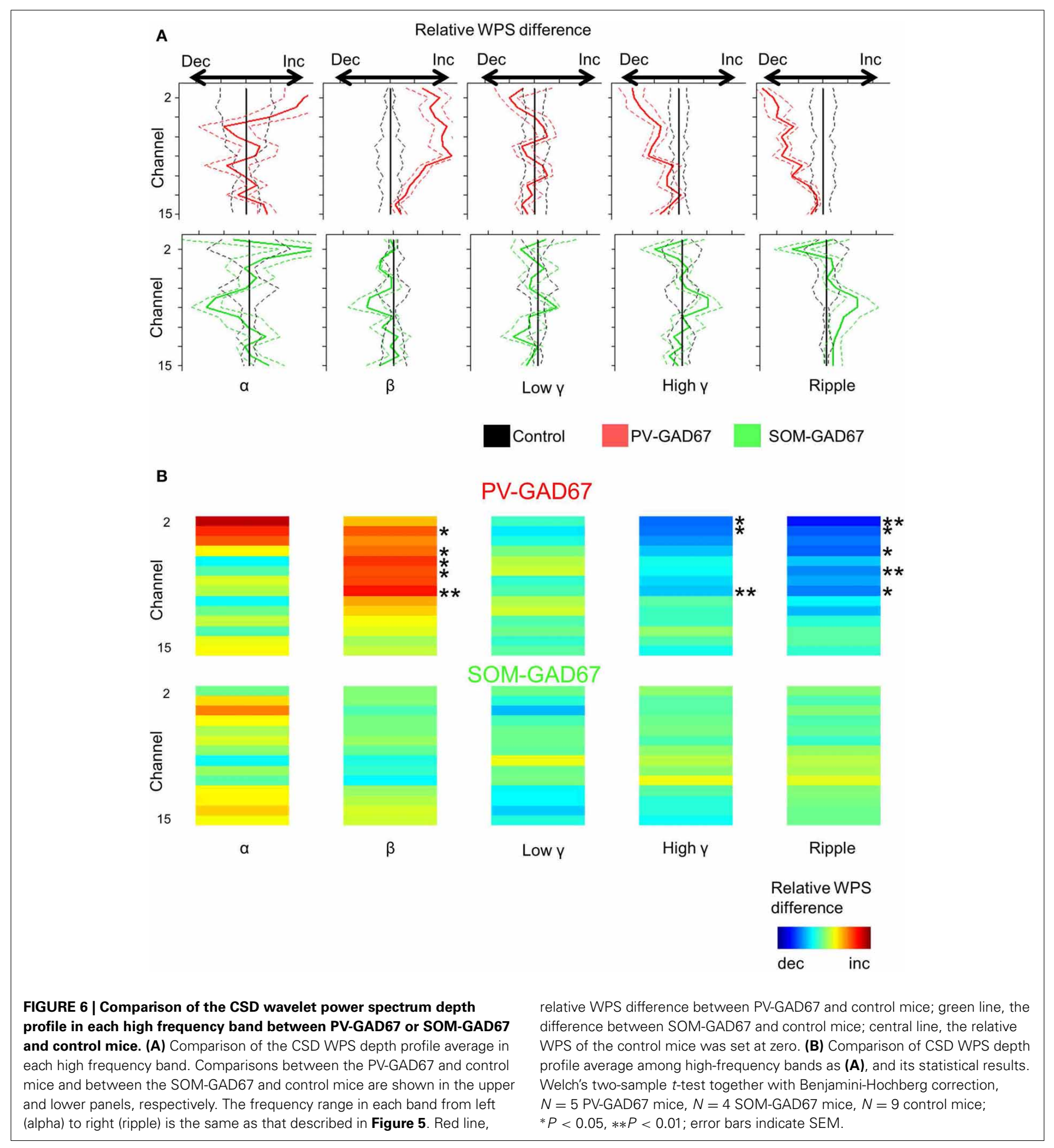

dendrites (Martinotti, 1889; Fairèn et al., 1984; Kawaguchi, 1993; Kawaguchi and Kubota, 1993; Condé et al., 1994; Hof et al., 1999; Tamamaki et al., 2003). It is important to generate horizontal propagation within pyramidal cells in layer 5 during the up state, which may be propagation among cell bodies of layer 5 pyramidal cells (Markram, 1997; Wester and Contreras, 2012; Beltramo et al., 2013). Therefore, inhibiting cell bodies by PV cells could be the main inhibitory system, whereas inhibiting distal dendrites by SOM cells could be a sub-inhibitory system. Decreases in PV cells activity disinhibit cell bodies of pyramidal cells in the absence of GAD67, and their hyperactivity activates $\mathrm{Ca}^{2+}$ - or $\mathrm{Na}^{+}$-dependent $\mathrm{K}^{+}$channels (i.e., activity-dependent $\mathrm{K}^{+}$channels) earlier, compared with control mice (Bazhenov et al., 2002; Compte et al., 2003; Hill and Tononi, 2005; Holcman 
and Tsodyks, 2006; Sanchez-Vives et al., 2010). Actually, our finding that MUA tended to increase in PV-GAD67 mice with a shortened up state was consistent with this interpretation. In contrast, SOM cell vacancy may have little effect on maintenance of the up state, during which distal input is not important. The CSD analysis also showed that the sink was concentrated in the deep layers during the up state, but not in the upper layers where distal dendrites gather. The sink is an inward current mainly caused by excitatory postsynaptic potentials (Mitzdorf, 1985). Therefore, our data strongly suggest that PV cells specifically contribute to persistence of the cortical up state.

The depth profiles of the high frequencies in PV-GAD67 mice were altered during up state of the slow oscillations; gamma and ripple oscillation power decreased in the neocortical upper layers, and beta oscillation power increased in the neocortical deep layers. We first considered how gamma and ripple power decreased in the upper layers of PV-GAD67 mice. The involvement of PV cells in gamma oscillations has been shown in several studies (Freund, 2003; Fuchs et al., 2007; Sohal et al., 2009; Buzsáki and Wang, 2012). Gamma oscillations are localized in the neocortical upper layer, whereas beta oscillations are localized in the neocortical deep layers (Roopun et al., 2006; Quilichini et al., 2010; Buffalo et al., 2011). The ripple oscillations observed in the neocortical up state are caused by fast-spiking interneurons, suggesting that gamma and ripple oscillations have similar mechanisms in the neocortex (Grenier et al., 2001). Although we treated high gamma and ripple oscillations separately, they could both be classified as high gamma oscillations. The decrease in gamma oscillations in the upper layers, as shown in PV-GAD67 mice, is consistent with these observations. Furthermore, patients with schizophrenia have reduced gamma oscillations, as well as reduced GAD67 expression (Gallinat et al., 2004; Akbarian and Huang, 2006). Indeed, a model study suggested that a reduction in the number of GAD67-expressing PV cells in patients with schizophrenia also reduces gamma oscillations (Volman et al., 2011). The PVGAD67 mice used in the current study showed loss of GAD67 in PV cells. Therefore, these results suggest that the decrease in gamma oscillations in patients with schizophrenia are related to loss of GAD67 in PV cells in vivo.

We next considered how deep beta power increased in PVGAD67 mice. According to a previous study, beta oscillations result from deep pyramidal cells connected via gap junctions, and they are enhanced by disinhibiting GABA(A) receptors (Roopun et al., 2006). Disturbing PV cells in the deep layers may enhance synchronization in the beta band by disinhibiting deep pyramidal cells. It has also been hypothesized that the triad of PV neurons, SOM neurons, and pyramidal cells is involved in beta oscillations (Vierling-Claassen et al., 2010; Li et al., 2013). Based on this hypothesis, disturbing PV cells may have enhanced beta oscillations by disinhibiting SOM cells. Our results suggest that PV cells are involved in balancing gamma and beta oscillations across layers in vivo.

We also found that delta oscillation power tended to increase in SOM-GAD67 mice, but tended to decrease in PV-GAD67 mice. Delta oscillations grouped as slow oscillations in the neocortex are assumed to be generated at two sites: neocortical delta and thalamic delta oscillations (Amzica and Steriade, 1998). However, inhibitory cell subtype involvement in delta oscillations has not been investigated. Furthermore, our data were limited to the neocortex. The relationships between delta oscillations and inhibitory cell subtypes will be studied in the future.

Our results showed a shortened up state, suggesting that PV cells maintain the up state and balance high-frequency oscillations across cortical layers. Based on the current findings and previous observations, we hypothesize that a series of events occurs from initiation to termination of the up state. Two possible mechanisms initiate the up state. The first is that spontaneous release of neurotransmitter activates pyramidal cells in the deep layers (Chauvette et al., 2010). The deep pyramidal cells play essential roles initiating and maintaining the up state (Sanchez-Vives and McCormick, 2000; Wester and Contreras, 2012; Beltramo et al., 2013). The second possibility is that the thalamus activates neocortical PV cells through spindle waves and initiates the up state. A group of fast-spiking interneurons fire early during the up state and phase lock to spindle waves from the thalamus (Puig et al., 2008; Ushimaru et al., 2012). Subsequently, PV cells in the upper layers are recruited to the up state by deep pyramidal cells or the thalamus, which initiate gamma oscillations and maintain the up state. Next, deep pyramidal cells maintain their activity via balanced suppression by PV cells. At the same time, gamma oscillations in the upper layers decrease gradually and beta oscillations increase reciprocally in the deep layers (Whittington et al., 1997; Faulkner et al., 1999; Olufsen et al., 2003). Finally, deep activity-dependent $\mathrm{K}^{+}$channels are activated gradually, subsequently terminating cellular activity (SanchezVives and McCormick, 2000; Bazhenov et al., 2002; Compte et al., 2003; Hill and Tononi, 2005; Holcman and Tsodyks, 2006; Sanchez-Vives et al., 2010). Our previous study also demonstrated that optogenetically stimulating the deep layers terminates the up state (Kuki et al., 2013). Therefore, PV cells not only balance highfrequency oscillations along the cortical layers, but they may also moderate cellular activity in the deep layers to maintain the up state.

Our results show that PV cells are involved in gamma-beta balance and up state maintenance. A question arises whether gamma-beta balance and up state maintenance are related. To answer this question, we conducted a statistical analysis of the relationship between beta-gamma balance and up state duration within each mice strain (data not shown). However, we found no significant correlations between them. Our findings suggest that high-frequency activity and slow oscillations are controlled independently.

Due to the limitations of our current study, we must consider long-term compensation in the knockout mice. We also have to consider the degree of contamination of PV cells undergoing Cre- mediated recombination in SOM-GAD67 mice. In SOMIRES-Cre line, which was used for the generation of SOM-GAD67 mice in this study, 6-10\% of neurons expressing a Cre-dependent reporter in any neocortical layers are fast-spiking/PV cells ( $\mathrm{Hu}$ et al., 2013). For example, the SOM-GAD67 mice showed moderate changes in slow oscillations compared with control mice. We did not find any changes in the hippocampus between knockout and control mice, whereas previous studies revealed that SOM and PV cells play roles during slow oscillations (Sun et al., 2002; 
Fanselow et al., 2008; Buzsáki and Wang, 2012). Loss of GABA(A) receptors in the cerebellum has little effect on normal neuronal behavior due to homeostatic plasticity (Brickley et al., 2001).

In conclusion, our results demonstrate that PV cells play particularly important roles maintaining the up state and balancing the gamma and beta bands across the cortical layers. SOM and $\mathrm{PV}$ cells may make asymmetric contributions to the regulation of up-state duration, firing rate during the up state, and regulating delta oscillations. This study was performed on anesthetized mice. It would be interesting to extend the study to behaving mice to understand how PV cells are related to cognitive function. In particular, the shortage of persistent cortical activity in PV-GAD67 mice may have been related to the insufficient cognition in schizophrenia. To overcome the problem of compensation and to understand the oscillatory mechanism more precisely, it would be interesting to manipulate PV and SOM cell activities in each layer selectively and transiently using optogenetics.

\section{AUTHORS AND CONTRIBUTORS}

Toshinobu Kuki, Hajime Mushiake, and Yuchio Yanagawa designed research. Toshinobu Kuki, Kazuyuki Fujihara, Hideki Miwa, and Nobuaki Tamamaki acquired data. Toshinobu Kuki analyzed data. Toshinobu Kuki and Hajime Mushiake interpreted data. Toshinobu Kuki drafted the paper. Hajime Mushiake, Yuchio Yanagawa, and Hideki Miwa revised the paper. Hajime Mushiake finally approved the version to be published. Hajime Mushiake agreed to be accountable for all aspects of the work.

\section{ACKNOWLEDGMENTS}

We thank Dr. Kunihiko Obata for providing GAD67-floxed mice and his encouragement. This study was supported by the Japan Science and Technology Agency (JST), Ministry of Education, Culture, Sports, Science and Technology Japan (MEXT), the Cooperative Program of the National Institute for Physiological Sciences, Japan, and the Takeda Science Foundation.

\section{REFERENCES}

Akbarian, S., and Huang, H. S. (2006). Molecular and cellular mechanisms of altered GAD1/GAD67 expression in schizophrenia and related disorders. Brain Res. Rev. 52, 293-304. doi: 10.1016/j.brainresrev.2006.04.001

Amzica, F., and Steriade, M. (1998). Electrophysiological correlates of sleep delta waves. Electroencephalogr. Clin. Neurophysiol. 107, 69-83. doi: 10.1016/S00134694(98)00051-0

Anderson, J., Lampl, I., Reichova, I., Carandini, M., and Ferster, D. (2000). Stimulus dependence of two state fluctuations of membrane potential in cat visual cortex. Nat. Neurosci. 3, 617-621. doi: 10.1038/75797

Bazhenov, M., Timofeev, I., Steriade, M., and Sejnowski, T. J. (2002). Model of thalamocortical slow wave sleep oscillations and transitions to activated states. J. Neurosci. 22, 8691-8704.

Beltramo, R., D’Urso, G., Dal Maschio, M., Farisello, P., Bovetti, S., Clovis, Y., et al. (2013). Layer specific excitatory circuits differentially control recurrent network dynamics in the neocortex. Nat. Neurosci. 16, 227-234. doi: 10.1038/nn.3306

Brickley, S. G., Revilla, V., Cull-Candy, S. G., Wisden, W., and Farrant, M. (2001). Adaptive regulation of neuronal excitability by a voltage-independent potassium conductance. Nature 409, 88-92. doi: 10.1038/35051086

Buffalo, E. A., Fries, P., Landman, R., Buschman, T. J., and Desimone, R. (2011). Laminar differences in gamma and alpha coherence in the ventral stream. Proc. Natl. Acad. Sci. U.S.A. 108, 11262-11267. doi: 10.1073/pnas.1011284108

Buzsáki, G., and Wang, X. J. (2012). Mechanisms of gamma oscillations. Annu. Rev. Neurosci. 35, 203-225. doi: 10.1146/annurev-neuro-062111-150444

Carlén, M., Meletis, K., Siegle, J. H., Cardin, J. A., Futai, K., Vierling-Claassen, D., et al. (2011). A critical role for NMDA receptors in parvalbumin interneurons for gamma rhythm induction and behavior. Mol. Psychiatr. 17, 537-548. doi: 10.1038/mp.2011.31

Chauvette, S., Volgushev, M., and Timofeev, I. (2010). Origin of active states in local neocortical networks during slow sleep oscillation. Cereb. Cortex 20, 2660-2674. doi: 10.1093/cercor/bhq009

Compte, A., Sanchez-Vives, M. V., McCormick, D. A., and Wang, X. J. (2003). Cellular and network mechanisms of slow oscillatory activity $(<1 \mathrm{~Hz})$ and wave propagations in a cortical network model. J. Neurophysiol. 89, 2707-2725. doi: 10.1152/jn.00845.2002

Condé, F., Lund, J. S., Jacobowitz, D. M., Baimbridge, K. G., and Lewis, D. A. (1994). Local circuit neurons immunoreactive for calretinin, calbindin D-28k or parvalbumin in monkey prefronatal cortex: distribution and morphology. J. Comp. Neurol. 341, 95-116. doi: 10.1002/cne.903410109

Destexhe, A., Contreras, D., and Steriade, M. (1999). Spatiotemporal analysis of local field potentials and unit discharges in cat cerebral cortex during natural wake and sleep states. J. Neurosci. 19, 4595-4608.

Fairèn, A., Javier, D., and José, R. (1984). Nonpyramidal neurons: general account. Cereb. Cortex 1, 201-253.

Fanselow, E. E., Richardson, K. A., and Connors, B. W. (2008). Selective, statedependent activation of somatostatin-expressing inhibitory interneurons in mouse neocortex. J. Neurophysiol. 100, 2640. doi: 10.1152/jn.90691.2008

Faulkner, H. J., Traub, R. D., and Whittington, M. A. (1999). Anaesthetic/amnesic agents disrupt beta frequency oscillations associated with potentiation of excitatory synaptic potentials in the rat hippocampal slice. Br. J. Pharmacol. 128, 1813-1825. doi: 10.1038/sj.bjp.0702948

Franklin, K. B., and Paxinos, G. (2008). The Mouse Brain in Stereotaxic Coordinates, Vol. 3. New York, NY: Academic press.

Freund, T. F. (2003). Interneuron diversity series: rhythm and mood in perisomatic inhibition. Trends Neurosci. 26, 489-495. doi: 10.1016/S0166-2236(03) 00227-3

Fuchs, E. C., Zivkovic, A. R., Cunningham, M. O., Middleton, S., LeBeau, F. E., Bannerman, D. M., et al. (2007). Recruitment of parvalbumin positive interneurons determines hippocampal function and associated behavior. Neuron 53, 591-604. doi: 10.1016/j.neuron.2007.01.031

Gallinat, J., Winterer, G., Herrmann, C. S., and Senkowski, D. (2004). Reduced oscillatory gamma-band responses in unmedicated schizophrenic patients indicate impaired frontal network processing. Clin. Neurophysiol. 115, 1863-1874. doi: 10.1016/j.clinph.2004.03.013

Gogolla, N., Takesian, A. E., Feng, G., Fagiolini, M., and Hensch, T. K. (2014). Sensory integration in mouse insular cortex reflects GABA circuit maturation. Neuron. 83, 894-905. doi: 10.1016/j.neuron.2014.06.033

Grenier, F., Timofeev, I., and Steriade, M. (2001). Focal synchronization of ripples $(80-200 \mathrm{~Hz})$ in neocortex and their neuronal correlates. J. Neurophysiol. 86, 1884-1898.

Hasenstaub, A., Shu, Y., Haider, B., Kraushaar, U., Duque, A., and McCormick, D. A. (2005). Inhibitory postsynaptic potentials carry synchronized frequency information in active cortical networks. Neuron 47, 423-435. doi: 10.1016/j.neuron.2005.06.016

Hill, S., and Tononi, G. (2005). Modeling sleep and wakefulness in the thalamocortical system. J. Neurophysiol. 93, 1671-1698. doi: 10.1152/jn.00915.2004

Hof, P. R., Glezer, I. I., Condé, F., Flagg, R. A., Rubin, M. B., Nimchinsky, E. A., et al. (1999). Cellular distribution of the calcium-binding proteins parvalbumin, calbindin, and calretinin in the neocortex of mammals: phylogenetic and developmental patterns. J. Chem. Neuroanat. 16, 77-116. doi: 10.1016/S08910618(98)00065-9

Holcman, D., and Tsodyks, M. (2006). The emergence of up and down states in cortical networks. PLoS Comput. Biol. 2:e23. doi: 10.1371/journal.pcbi.0020023

Hu, H., Cavendish, J. Z., and Agmon, A. (2013). Not all that glitters is gold: off-target recombination in the somatostatin-IRES-Cre mouse line labels a subset of fast-spiking interneurons. Front. Neural Circuits 7:195. doi: 10.3389/fncir.2013.00195

Kawaguchi, Y. (1993). Groupings of nonpyramidal and pyramidal cells with specific physiological and morphological characteristics in rat frontal cortex. J. Neurophysiol. 69, 416-416.

Kawaguchi, Y., and Kubota, Y. (1993). Correlation of physiological subgroupings of nonpyramidal cells with parvalbumin-and calbindinD28k-immunoreactive neurons in layer V of rat frontal cortex. J. Neurophysiol. 70, 387-396.

Kitano, K., Cateau, H., Kaneda, K., Nambu, A., Takada, M., and Fukai, T. (2002). Two-state membrane potential transitions of striatal spiny neurons as evidenced 
by numerical simulations and electrophysiological recordings in awake monkeys. J. Neurosci. 22: RC230.

Kuki, T., Ohshiro, T., Ito, S., Ji, Z. G., Fukazawa, Y., Matsuzaka, Y., et al. (2013). Frequency dependent entrainment of neocortical slow oscillation to repeated optogenetic stimulation in the anesthetized rat. Neurosci. Res. 75, 35-45. doi: 10.1016/j.neures.2012.10.007

Lakatos, P., Shah, A. S., Knuth, K. H., Ulbert, I., Karmos, G., and Schroeder, C. E. (2005). An oscillatory hierarchy controlling neuronal excitability and stimulus processing in the auditory cortex. J. Neurophysiol. 94, 1904-1911. doi: 10.1152/jn.00263.2005

Le Bon-Jego, M., and Yuste, R. (2007). Persistently active, pacemaker-like neurons in neocortex. Front. Neurosci. 1:123. doi: 10.3389/neuro.01.1.1. 009.2007

Li, X., Morita, K., Robinson, H. P., and Small, M. (2013). Control of layer 5 pyramidal cell spiking by oscillatory inhibition in the distal apical dendrites: a computational modeling study. J. Neurophysiol. 109, 2739-2756. doi: 10.1152/jn.00397.2012

Lisman, J. E., Coyle, J. T., Green, R. W., Javitt, D. C., Benes, F. M., Heckers, S., et al. (2008). Circuit-based framework for understanding neurotransmitter and risk gene interactions in schizophrenia. Trends Neurosci. 31, 234-242. doi: 10.1016/j.tins.2008.02.005

Maier, A., Aura, C. J., and Leopold, D. A. (2011). Infragranular sources of sustained local field potential responses in macaque primary visual cortex. J. Neurosci. 31, 1971-1980. doi: 10.1523/JNEUROSCI.5300-09.2011

Mann, E. O., Kohl, M. M., and Paulsen, O. (2009). Distinct roles of $\mathrm{GABA}_{A}$ and $\mathrm{GABA}_{B}$ receptors in balancing and terminating persistent cortical activity. J. Neurosci. 29, 7513-7518. doi: 10.1523/JNEUROSCI.6162-08.2009

Markram, H. (1997). A network of tufted layer 5 pyramidal neurons. Cereb. Cortex 7, 523-533. doi: 10.1093/cercor/7.6.523

Martinotti, C. (1889). Contributo allo studio della corteccia cerebrale, ed all'origine centrale dei nervi. Ann. Freniatr. Sci. Affini. 1, 14-381.

Mitzdorf, U. (1985). Current source-density method and application in cat cerebral cortex: investigation of evoked potentials and EEG phenomena. Am. Physiol. Soc. 65, 37-90.

Mitzdorf, U., and Singer, W. (1977). Laminar segregation of afferents to lateral geniculate nucleus of the cat: an analysis of current source density. J. Neurophysiol. 40, 1227-1244.

Mizuhara, H., and Yamaguchi, Y. (2011). Neuronal ensemble for visual working memory via interplay of slow and fast oscillations. Eur. J. Neurosci. 33, 1925-1934. doi: 10.1111/j.1460-9568.2011.07681.x

Nicholson, C., and Freeman, J. A. (1975). Theory of current source-density analysis and determination of conductivity tensor for anuran cerebellum. J. Neurophysiol. 38, 356-368.

Obata, K., Hirono, M., Kume, N., Kawaguchi,Y., Itohara, S., and Yanagawa, Y. (2008). GABA and synaptic inhibition of mouse cerebellum lacking glutamate decarboxylase 67. Biochem. Biophys. Res. Commun. 370, 429-433. doi: 10.1016/j.bbrc.2008.03.110

Olufsen, M. S., Whittington, M. A., Camperi, M., and Kopell, N. (2003). New roles for the gamma rhythm: population tuning and preprocessing for the beta rhythm. J. Comput. Neurosci. 14, 33-54. doi: 10.1023/A:1021124 317706

Puig, M. V., Ushimaru, M., and Kawaguchi, Y. (2008). Two distinct activity patterns of fast-spiking interneurons during neocortical UP states. Proc. Natl. Acad. Sci. U.S.A. 105, 8428-8433. doi: 10.1073/pnas.0712219105

Quilichini, P., Sirota, A., and Buzsaki, G. (2010). Intrinsic circuit organization and theta-gamma oscillation dynamics in the entorhinal cortex of the rat. J. Neurosci. 30, 11128-11142. doi: 10.1523/JNEUROSCI.1327-10.2010

Rappelsberger, P., Pockberger, H., and Petsche, H. (1981). Current source density analysis: methods and application to simultaneously recorded field potentials of the rabbit's visual cortex. Pflügers Arch. 389, 159-170. doi: 10.1007/BF00 582108

Roopun, A. K., Middleton, S. J., Cunningham, M. O., LeBeau, F. E. N., Bibbig, A., Whittington, M. A., et al. (2006). A beta2-frequency $(20-30 \mathrm{~Hz})$ oscillation in nonsynaptic networks of somatosensory cortex. Proc. Natl. Acad. Sci. U.S.A. 103, 15646-15650. doi: 10.1073/pnas.0607443103

Ruiz-Mejias, M., Ciria-Suarez, L., Mattia, M., and Sanchez-Vives, M. V. (2011). Slow and fast rhythms generated in the cerebral cortex of the anesthetized mouse. J. Neurophysiol. 106, 2910-2921. doi: 10.1152/jn.00440.2011
Sanchez-Vives, M. V., Mattia, M., Compte, A., Perez-Zabalza, M., Winograd, M., Descalzo, V. F., et al. (2010). Inhibitory modulation of cortical up states. J. Neurophysiol. 104, 1314-1324. doi: 10.1152/jn. 00178.2010

Sanchez-Vives, M. V., and McCormick, D. A. (2000). Cellular and network mechanisms of rhythmic recurrent activity in neocortex. Nat. Neurosci. 3, 1027-1034. doi: 10.1038/79848

Sharma, A. V., Wolansky, T., and Dickson, C. T. (2010). A comparison of sleeplike slow oscillations in the hippocampus under ketamine and urethane anesthesia. J. Neurophysiol. 104, 932-939. doi: 10.1152/jn. 01065.2009

Sohal, V. S., Zhang, F., Yizhar, O., and Deisseroth, K. (2009). Parvalbumin neurons and gamma rhythms enhance cortical circuit performance. Nature 459, 698-702. doi: 10.1038/nature07991

Somogyi, P., and Cowey, A. (1984). Double bouquet cells. Cereb. Cortex 1, 337-360. Steriade, M., Contreras, D., Amzica, F., and Timofeev, I. (1996). Synchronization of fast $(30-40 \mathrm{~Hz})$ spontaneous oscillations in intrathalamic and thalamocortical networks. J. Neurosci. 16, 2788-2808.

Steriade, M., McCormick, D. A., and Sejnowski, T. J. (1993c). Thalamocortical oscillations in the sleeping and aroused brain. Science 262, 679-685. doi: $10.1126 /$ science. 8235588

Steriade, M., Nunez, A., and Amzica, F. (1993a). A novel slow $(<1 \mathrm{~Hz})$ oscillation of neocortical neurons in vivo: depolarizing and hyperpolarizing components. J. Neurosci. 13, 3252-3265.

Steriade, M., Nunez, A., and Amzica, F. (1993b). Intracellular analysis of relations between the slow $(<1 \mathrm{~Hz})$ neocortical oscillation and other sleep rhythms of the electroencephalogram. J. Neurosci. 13, 3266-3283.

Sun, Q. Q., Huguenard, J. R., and Prince, D. A. (2002). Somatostatin inhibits thalamic network oscillations in vitro: actions on the GABAergic neurons of the reticular nucleus. J. Neurosci. 22, 5374-5386.

Tamamaki, N., Yanagawa, Y., Tomioka, R., Miyazaki, J. I., Obata, K., and Kaneko, T. (2003). Green fluorescent protein expression and colocalization with calretinin, parvalbumin, and somatostatin in the GAD67GFP knock-in mouse. J. Comp. Neurol. 467, 60-79. doi: 10.1002/cne. 10905

Tanahira, C., Higo, S., Watanabe, K., Tomioka, R., Ebihara, S., Kaneko, T., et al. (2009). Parvalbumin neurons in the forebrain as revealed by parvalbuminCre transgenic mice. Neurosci. Res. 63, 213-223. doi: 10.1016/j.neures.2008. 12.007

Taniguchi, H., He, M., Wu, P., Kim, S., Paik, R., Sugino, K., et al. (2011). A resource of Cre driver lines for genetic targeting of GABAergic neurons in cerebral cortex. Neuron 71, 995-1013. doi: 10.1016/j.neuron.2011. 07.026

Timofeev, I., Grenier, F., Bazhenov, M., Sejnowski, T. J., and Steriade, M. (2000). Origin of slow cortical oscillations in deafferented cortical slabs. Cereb. Cortex 10, 1185-1199. doi: 10.1093/cercor/10.12.1185

Ushimaru, M., Ueta, Y., and Kawaguchi, Y. (2012). Differentiated participation of thalamocortical subnetworks in slow/spindle waves and desynchronization. J. Neurosci. 32, 1730-1746. doi: 10.1523/JNEUROSCI.4883-11.2012

Vierling-Claassen, D., Cardin, J. A., Moore, C. I., and Jones, S. R. (2010). Computational modeling of distinct neocortical oscillations driven by celltype selective optogenetic drive: separable resonant circuits controlled by lowthreshold spiking and fast-spiking interneurons. Front. Hum. Neurosci. 4:198. doi: 10.3389/fnhum.2010.00198

Volman, V., Behrens, M. M., and Sejnowski, T. J. (2011). Downregulation of parvalbumin at cortical GABA synapses reduces network gamma oscillatory activity. J. Neurosci. 31, 18137-18148. doi: 10.1523/JNEUROSCI.304111.2011

Wester, J. C., and Contreras, D. (2012). Columnar interactions determine horizontal propagation of recurrent network activity in neocortex. J. Neurosci. 32, 5454-5471. doi: 10.1523/JNEUROSCI.5006-11.2012

Whittington, M. A., Traub, R. D., Faulkner, H. J., Stanford, I. M., and Jefferys, J. G. (1997). Recurrent excitatory postsynaptic potentials induced by synchronized fast cortical oscillations. Proc. Natl. Acad. Sci. U.S.A. 94, 12198-12203. doi: 10.1073/pnas.94.22.12198

Wilson, C. J., and Kawaguchi, Y. (1996). The origins of two-state spontaneous membrane potential fluctuations of neostriatal spiny neurons. J. Neurosci. 16, 2397-2410. 
Wolansky, T., Clement, E. A., Peters, S. R., Palczak, M. A., and Dickson, C. T. (2006). Hippocampal slow oscillation: a novel EEG state and its coordination with ongoing neocortical activity. J. Neurosci. 26, 6213-6229. doi: 10.1523/JNEUROSCI.5594-05.2006

Conflict of Interest Statement: The authors declare that the research was conducted in the absence of any commercial or financial relationships that could be construed as a potential conflict of interest.

Received: 15 October 2014; paper pending published: 18 December 2014; accepted: 14 January 2015; published online: 03 February 2015.
Citation: Kuki T, Fujihara K, Miwa H, Tamamaki N, Yanagawa Y and Mushiake H (2015) Contribution of parvalbumin and somatostatin-expressing GABAergic neurons to slow oscillations and the balance in beta-gamma oscillations across cortical layers. Front. Neural Circuits 9:6. doi: 10.3389/fncir.2015.00006

This article was submitted to the journal Frontiers in Neural Circuits.

Copyright (c) 2015 Kuki, Fujihara, Miwa, Tamamaki, Yanagawa and Mushiake. This is an open-access article distributed under the terms of the Creative Commons Attribution License (CC BY). The use, distribution or reproduction in other forums is permitted, provided the original author(s) or licensor are credited and that the original publication in this journal is cited, in accordance with accepted academic practice. No use, distribution or reproduction is permitted which does not comply with these terms. 